\title{
On the Chromatic Number of some Flip Graphs
}

\author{
Ruy Fabila-Monroy ${ }^{1}$, David Flores-Peñaloza ${ }^{1}$, Clemens Huemer $\left.^{2}\right|^{\mid}$

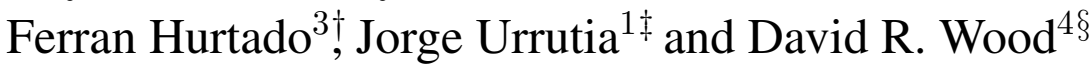 \\ ${ }^{1}$ Instituto de Matemáticas, Universidad Nacional Autónoma de México \\ ruy@ciencias.unam.mx, \{dflores, urrutia\} emath.unam.mx \\ ${ }^{2}$ Departament de Matemàtica Aplicada IV, Universitat Politècnica de Catalunya, Barcelona, Spain \\ clemens@ma4.upc.edu \\ ${ }^{3}$ Departament de Matemàtica Aplicada II, Universitat Politècnica de Catalunya, Barcelona, Spain \\ ferran.hurtado@upc.edu \\ ${ }^{4}$ Department of Mathematics and Statistics, The University of Melbourne, Melbourne, Australia \\ wooddeunimelb.edu. au
}

received September 12, 2008, revised May 26, 2009, accepted June 16, 2009.

This paper studies the chromatic number of the following four flip graphs (under suitable definitions of a flip):

- the flip graph of perfect matchings of a complete graph of even order,

- the flip graph of triangulations of a convex polygon (the associahedron),

- the flip graph of non-crossing Hamiltonian paths of a set of points in convex position, and

- the flip graph of triangles in a convex point set.

We give tight bounds for the latter two cases and upper bounds for the first two.

Keywords: chromatic number, flip graph, geometric graph, Hamiltonian path, perfect matching, triangulation

Mathematics Subject Classification: 05C15 (Coloring of graphs and hypergraphs)

\footnotetext{
${ }^{\dagger}$ Research of C. Huemer and F. Hurtado supported by projects MEC MTM2006-01267 and Gen. Cat. 2005SGR00692.

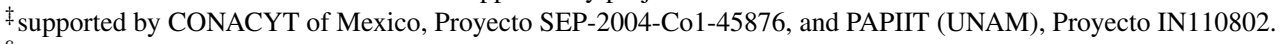

$\S$ Supported by a QEII Research Fellowship. Research initiated at the Universitat Politècnica de Catalunya, where supported by a Marie Curie Fellowship under contract MEIF-CT-2006-023865, and by projects MEC MTM2006-01267 and DURSI 2005SGR00692.
}

1365-8050 @ 2009 Discrete Mathematics and Theoretical Computer Science (DMTCS), Nancy, France 


\section{Introduction}

Given a class $\mathcal{C}$ of combinatorial objects of a given kind and a transformation (flip) between these objects, a flip graph is defined as the graph whose vertex set is $\mathcal{C}$, where two vertices are adjacent whenever the corresponding objects differ by a flip. Flip graphs have received considerable attention in the past. Properties such as Hamiltonicity, connectivity and diameter have been widely studied [4, 6, 13, 16]. This interest is very likely a consequence of the practical applications of these properties. For example, Hamiltonicity allows for rapid generation of the given combinatorial objects. We refer the interested reader to the survey [1].

A $k$-coloring of a graph $G$ is a function that assigns one of $k$ colors to each vertex of $G$, such that adjacent vertices receive distinct colors. The chromatic number $\chi(G)$ is the least integer $k$ for which there is a $k$-coloring of $G$. The chromatic number can be formulated in terms of homomorphisms. A homomorphism from a graph $G$ to a graph $H$ is a mapping from $V(G)$ to $V(H)$ such that the images of any two adjacent vertices in $G$ are adjacent in $H$. In this sense $\chi(G)$ is the minimum positive integer $m$ such that there exists a homomorphism from $G$ to the complete graph $K_{m}$. The composition of homomorphisms is again a homomorphism. Therefore if there exists a homomorphism from $G$ to $H$ then $\chi(G) \leq \chi(H)$.

The chromatic number of flip graphs has received little attention, with only a few papers concentrating on this parameter (see for example [5]). This paper studies the chromatic number of some flip graphs. We first consider three flip graphs defined on sets of points in convex position. (A set of points in the plane is in convex position if it forms the vertex-set of a convex polygon.) We then consider the flip graph on the perfect matchings of a complete graph of even order.

A geometric non-crossing graph is a planar graph in which the vertices are embedded as points in the plane, and where the edges are embedded as non-crossing straight line segments. Throughout the paper, $S_{n}$ will denote a set of $n$ points in convex position in the plane, and the term convex hull will refer to the boundary of the convex polygon having those points as vertex set. All of the considered graphs, except the flip graph of perfect matchings, have all of their vertices associated to non-crossing geometric graphs having $S_{n}$ as vertex set. We remark that any underlying convex point set of the same size induces the same graphs.

This paper is organized as follows. In Section 2 we determine the exact chromatic number for noncrossing geometric Hamiltonian paths having $S_{n}$ as their set of vertices. In Section 3 we define a certain flip graph on the triangles with vertices in $S_{n}$ and determine its chromatic number up to a constant multiplicative factor. In Section 4 we give an upper bound on the chromatic number for the flip graph of the triangulations of $S_{n}$. In Section 5 we consider a flip graph on the perfect matchings of $K_{2 n}$ and give an upper bound on its chromatic number. (It should be stressed that in this graph no geometry is considered.) In Section 6 we conclude with some open problems and remarks.

\section{Geometric Non-Crossing Hamiltonian Paths}

Let $G_{n}$ be the graph whose vertex set is the set of all geometric non-crossing paths with vertex set $S_{n}$. Two paths $\Gamma$ and $\Gamma^{\prime}$ in $V\left(G_{n}\right)$ are adjacent if and only if $\Gamma^{\prime}$ is obtained from $\Gamma$ by deleting some edge $e$ and adding some edge $f$. (See Figure 1) We say that $\Gamma^{\prime}$ is obtained from $\Gamma$ by flipping the edge $e$. We point out that $e$ and $f$ may cross or they may share an endpoint. Rivera-Campo [15] and Urrutia-Galicia [17] proved that $G_{n}$ is Hamiltonian. We determine $\chi\left(G_{n}\right)$.

Theorem 2.1 For every integer $n \geq 3, \chi\left(G_{n}\right)=n$. 


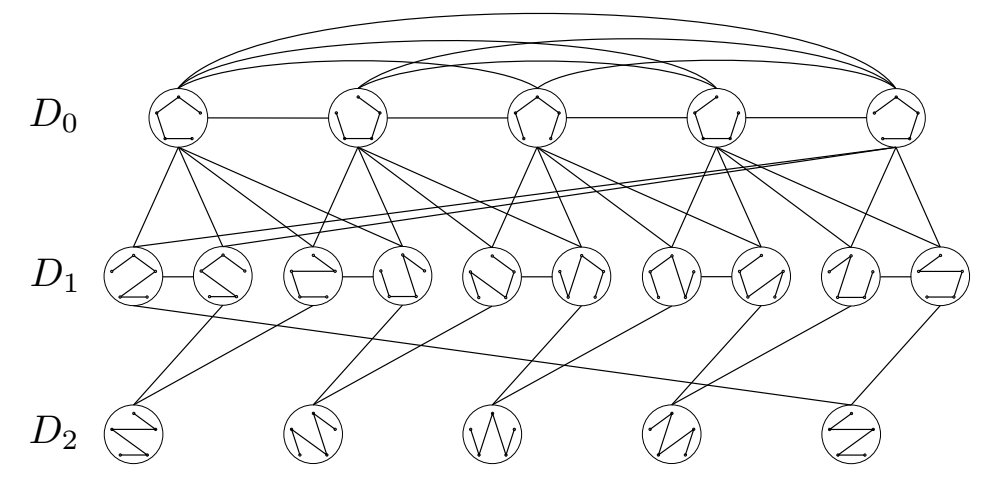

Fig. 1: $G_{5}$ drawn with vertices grouped by the number of non-convex hull edges.

Proof: Since $G_{3} \simeq K_{3}$, assume $n \geq 4$. For all integers $i \in[0, n-3]$, let $D_{i}$ be the set of paths in $V\left(G_{n}\right)$ that contain exactly $i$ edges not in the convex hull of $S_{n}$. (See Figure 1). Note that the set $D_{0}$ consists of $n$ paths, all of whose edges are in the convex hull of $S_{n}$. Each element of $D_{0}$ is obtained by removing one edge of the convex hull of $S_{n}$. Clearly any two elements of $D_{0}$ are adjacent in $G_{n}$, therefore $D_{0}$ induces a complete subgraph of $G_{n}$. This proves that $\chi\left(G_{n}\right) \geq n$.

Let $H_{i}$ be the subgraph induced in $G_{n}$ by $D_{0} \cup \cdots \cup D_{i}$. We prove $\chi\left(G_{n}\right) \leq n$ by showing how to extend an $n$-coloring of $H_{i-1}$ to an $n$-coloring of $H_{i}$ for every $i \in[1, n-3]$. For this, let $\Gamma_{i}$ be a path in $D_{i}$.

For extending an $n$-coloring of $H_{0}$ to one of $H_{1}$ we use the next two properties of $G_{n}$. (1) The set $D_{1}$ induces a perfect matching in $G_{n}$. (2) Every adjacent pair in $D_{1}$ together with its neighborhood in $H_{1}$ induces a $K_{4}$ in $G_{n}$. Both properties follow because, on one hand, flipping an edge of $\Gamma_{1}$ that is in the convex hull of $S_{n}$ yields a path in $D_{2}$. On the other hand, flipping the edge of $\Gamma_{1}$ that is not in the convex hull of $S_{n}$ yields either one path in $D_{1}$, or one of two different paths in $D_{0}$. Since $n \geq 4$, each pair of adjacent paths in $D_{1}$ can be colored with colors different to those of its neighbors in $D_{0}$.

For extending an $n$-coloring of $H_{i-1}$ to one of $H_{i}$ when $i \geq 2$, we use the next two properties of $G_{n}$. (1) The set $D_{i}$ is an independent set in $G_{n}$. (2) The vertex of $G_{n}$ corresponding to the path $\Gamma_{i}$ has degree 2 in $H_{i}$. The first property follows because, on one hand, flipping an edge of $\Gamma_{i}$ that $i s$ in the convex hull of $S_{n}$ yields a path in $D_{i+1}$. On the other hand, flipping an edge of $\Gamma$ that is not in the convex hull of $S_{n}$ yields a path in $D_{i-1}$. The latter property follows because the only edges of $\Gamma$ that are not in the convex hull of $S_{n}$ that can be flipped, are the first and last when walking from one leaf of $\Gamma$ to the other.

Since $n$ colors are needed to color $D_{0}$, and any $n$-coloring of $D_{0}$ can be extended to an $n$-coloring of $G_{n}$, the result follows.

\section{Triangle Graph}

We introduce a new flip graph $G_{\triangle}(n)$, whose vertex set is the set of triangles with endpoints in $S_{n}$, two of which are adjacent if they share an edge and their interiors are disjoint. (See Figure 2) We prove that this graph has chromatic number $\Theta(\log n)$. 


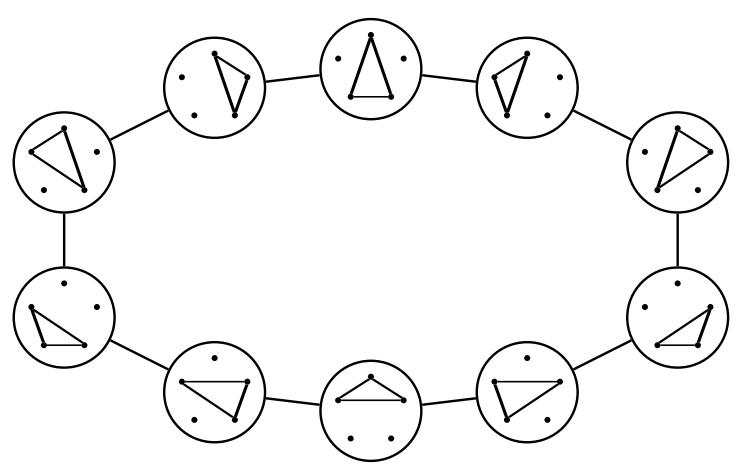

Fig. 2: The graph $G_{\triangle}(10)$.

For the rest of this section, assume that the elements of $S_{n}$ are labeled $\{0, \ldots, n-1\}$ in clockwise order, and let any triangle be denoted by the set of its vertices.

Lemma 3.1 For every integer $n \geq 3, \chi\left(G_{\triangle}(n)\right) \geq \log _{2}(n-1)$.

Proof: Let $H$ be the subgraph of $G_{\triangle}(n)$ induced by all triangles containing 0 as a vertex. Suppose that $H$ has a $k$-coloring. Let $A_{i}$ (with $1 \leq i \leq n-2$ ), be the set of colors assigned to the triangles $\{0, i, j\}$ (with $i<j \leq n-1$ ); see Figure 3 (a). Observe that $A_{r} \neq A_{s}$ for $r \neq s$. This follows from the fact that if $r<s$, the triangle $\{0, r, s\}$ is adjacent to every triangle $\{0, s, k\}$ (with $s<k \leq n-1$ ); see Figure 3 (b). Thus, the color assigned to $\{0, r, s\}$ is in $A_{r}$ but not in $A_{s}$. The number of possible non-empty color subsets must be at least $n-2$. Therefore $2^{k} \geq n-1$, implying $k \geq \log _{2}(n-1)$ and $\chi\left(G_{\triangle}(n)\right) \geq \log _{2}(n-1)$.

Note that the subgraph $H$ of $G_{\triangle}$ considered in the proof of Lemma 3.1 is isomorphic to the so-called shift graph [7] of the set $\{1,2, \ldots, n-1\}$. It is folklore that its chromatic number is $\left\lceil\log _{2}(n-1)\right\rceil$.

Lemma 3.2 For every integer $m \geq 3$, let $G$ be the subgraph of $G_{\triangle}(2 m)$ induced by all the triangles without edges in the convex hull of $S_{2 m}$. Then $\chi(G)=\chi\left(G_{\triangle}(m)\right)$.

Proof: Color the vertices of $S_{2 m}$ red and blue such that consecutive vertices along the convex hull have distinct colors. Note that the subgraph $H$ of $G$ induced by the blue points is isomorphic to $G_{\triangle}(m)$. Map every triangle $t$ in $V(G)$ to a triangle $t^{\prime}$ in $V(H)$ as follows: If $i$ is a red vertex of $t$, substitute it by the blue vertex $i+1$, where addition is modulo $2 m$. Observe that triangles whose vertices are all blue are mapped onto themselves. Since adjacent triangles in $G$ are mapped to adjacent triangles in $H$, this mapping induces a homomorphism from $G$ to $H$ (see Figure 3). The result follows.

Lemma 3.3 For every integer $m \geq 3, \chi\left(G_{\triangle}(2 m)\right) \leq \chi\left(G_{\triangle}(m)\right)+3$.

Proof: We use a similar technique to that used in Theorem 2.1. Let $G^{\prime}$ be the subgraph of $G_{\triangle}(2 m)$ induced by all those triangles of $S_{2 m}$ that have at least one edge in the convex hull of $S_{2 m}$. Let $\tau=$ $\{i, i+1, j\}$ be any such triangle. $S_{2 m}-\{i, i+1, j\}$ can be partitioned into two maximal subsets of 


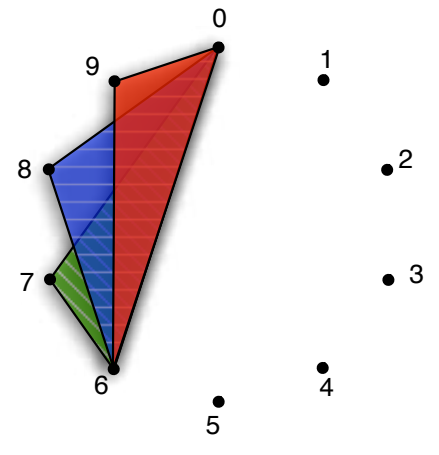

(a)

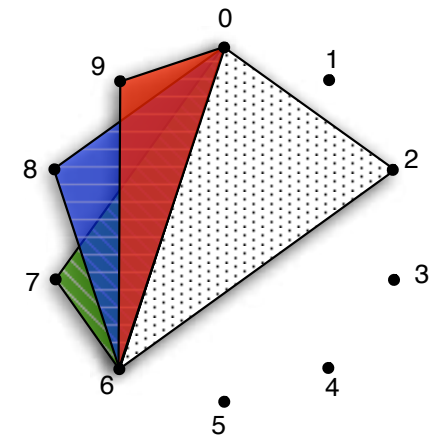

(b)

Fig. 3: Illustration of Lemma 3.1 (with $s=6$ ). (a) The triangles considered in $A_{s}$ are those having the segment $\overline{0 s}$ as its right side. (b) The triangle $\{0, r, s\}$ (with $r<s$ ) is adjacent in $H$ to all the triangles considered in $A_{s}$.

consecutive points along the convex hull, namely the points from $i+1$ to $j$ and the points from $j$ to $i$, which we denote by $l_{\tau}$ and $r_{\tau}$ respectively. Let the $\operatorname{order}$ of $\tau$ be $\min \left\{\left|l_{\tau}\right|,\left|r_{\tau}\right|\right\}$. For $i \in[0, m-2]$, let $D_{i}$ be the subset of $V\left(G^{\prime}\right)$ of all triangles of order $i$. Note that the subgraph of $G^{\prime}$ induced by $D_{m-2}$ has maximum degree 2 and therefore chromatic number at most 3 . Note that every vertex of $D_{i}$ is only adjacent to at most two vertices in $D_{i} \cup \cdots \cup D_{m-2}$. Thus any 3-coloring of $D_{i+1} \cup \cdots \cup D_{m-2}$ can be extended to a 3-coloring of $D_{i} \cup D_{i+1} \cup \cdots \cup D_{m-2}$. Therefore $\chi\left(G^{\prime}\right)=3$.

Let $G$ be the subgraph of $G_{\triangle}(2 m)$ induced by all the triangles without edges of the convex hull of $S_{2 m}$. Observe that $V(G) \cup V\left(G^{\prime}\right)=V\left(G_{\triangle}(2 m)\right)$. Since there is a 3-coloring of $G^{\prime}$ and (by Lemma 3.2) there is a coloring of $G$ with $\chi\left(G_{\triangle}(m)\right)$ colors, there is a coloring of $G_{\triangle}(2 m)$ with $\chi\left(G_{\triangle}(m)\right)+3$ colors.

We now have:

Theorem 3.4 For every integer $n \geq 3, \log _{2}(n-1) \leq \chi\left(G_{\triangle}(n)\right) \leq 3\left\lceil\log _{2}(n)\right\rceil-4$.

Proof: Let $F(k)=\chi\left(G_{\triangle}\left(2^{k}\right)\right)$. By Lemma 3.3. $F(k) \leq F(k-1)+3$. Observe that $\chi\left(G_{\triangle}(4)\right)=2$. Thus $F(k) \leq 3 k-4$ and therefore $\chi\left(G_{\triangle}\left(2^{k}\right)\right) \leq 3 k-4$.

For $n \neq 2^{k}$, let $m$ be the smallest power of 2 greater than $n$. Since $G_{\triangle}(n)$ is a subgraph of $G_{\triangle}(m)$, we can color the vertices of $G_{\triangle}(n)$ with $\chi\left(G_{\triangle}(m)\right) \leq 3 \log _{2}(m)-4=3\left\lceil\log _{2}(n)\right\rceil-4$ colors.

\section{Triangulations of a Convex Polygon}

We consider a flip graph on the triangulations of $S_{n}$. Let $G_{T}(n)$ be the graph whose vertex set is the set of triangulations of $S_{n}$, where two triangulations $\mathcal{T}_{1}$ and $\mathcal{T}_{2}$ are adjacent in $G_{T}(n)$ whenever they differ by one edge flip; that is, there exist edges $e \in \mathcal{T}_{1}$ and $f \in \mathcal{T}_{2}$ such that $\mathcal{T}_{2}$ is obtained from $\mathcal{T}_{1}$ by deleting $e$ and adding $f$ (see Figure 5p. 


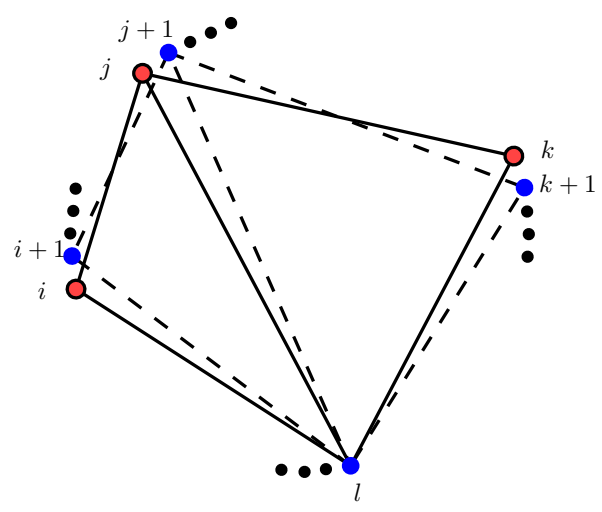

Fig. 4: The homomorphism between $G$ and $G \triangle(m)$.

Much is known about $G_{T}(n)$. This is probably because there is a bijection between triangulations of the $n$-gon and binary trees with $n-2$ interior nodes. A flip in a triangulation corresponds to a rotation in its corresponding binary tree. Lucas [13] proved that $G_{T}(n)$ is Hamiltonian. Sleator et al. [16] proved that the diameter of $G_{T}(n)$ is $2 n-10$. Lee [11] proved that the automorphism group of $G_{T}(n)$ is the dihedral group of symmetries of a regular $n$-gon. Lee also proved that $G_{T}(n)$ can be realized as the skeleton of an $(n-3)$-dimensional convex polytope called the associahedron. Most of these results are proved again in [10] using a unifying framework called the tree of triangulations.
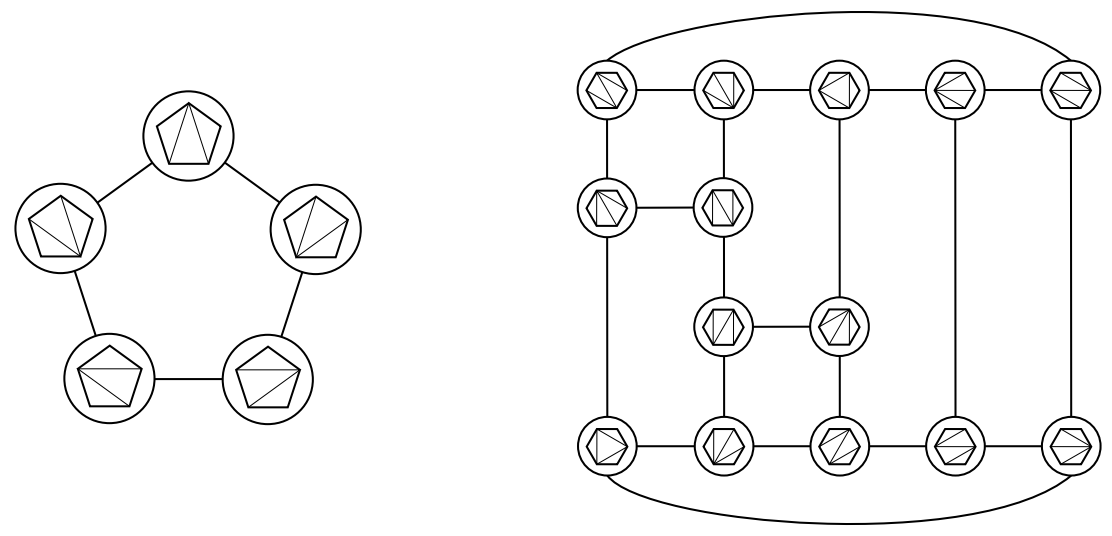

Fig. 5: The graphs $G_{T}(5)$ and $G_{T}(6)$.

We give an upper bound on the chromatic number of $G_{T}(n)$.

Theorem 4.1 For every integer $n \geq 3, \chi\left(G_{T}(n)\right) \leq\left\lceil\frac{n}{2}\right\rceil$.

Proof: It is well known that for $n$ even, the set of $\left(\begin{array}{c}n \\ 2\end{array}\right)$ edges between the vertices of $S_{n}$ can be partitioned into $\frac{n}{2}$ edge-disjoint geometric non-crossing paths (see [12, 2] and Figure 6 for an example with $n=6$ ). 
Label these paths $P_{1}, \ldots, P_{\frac{n}{2}}$. (Since $G_{T}(n)$ is a subgraph of $G_{T}(n+1)$, assume $n$ is even for the time being.) If an edge $e$ belongs to $P_{i}$, assign to it the weight $w(e)=i$. To every triangulation $\mathcal{T}$ of $S_{n}$, assign the number $\left(\sum_{e \in \mathcal{T}} w(e)\right) \bmod \frac{n}{2}$. Let $\mathcal{T}_{1}$ and $\mathcal{T}_{2}$ be two adjacent triangulations of $G_{T}(n)$. That is, $\mathcal{T}_{2}=\mathcal{T}_{1}-e+f$ for some crossing edges $e$ and $f$. Since $e$ and $f$ cross, $w(e) \neq w(f)$, and thus the numbers associated to $\mathcal{T}_{1}$ and $\mathcal{T}_{2}$ are different. This induces a coloring of $G_{T}(n)$ with $\frac{n}{2}$ colors for $n$ even and $\left\lceil\frac{n}{2}\right\rceil$ in general.

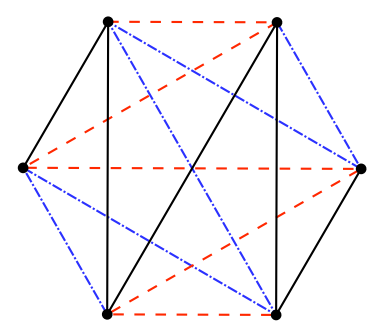

Fig. 6: Partition of the edges into disjoint non-crossing geometric Hamiltonian paths.

The upper bound on $\chi\left(G_{T}(n)\right)$ in Theorem 4.1 is non-trivial since, for example, Brooks' Theorem [3] gives an upper bound of only $n-3$. However, $\chi\left(G_{T}(n)\right)$ is in fact sublinear, as we now show. Johansson proved that for sufficiently large $\Delta$, every triangle-free graph with maximum degree $\Delta$ is $O\left(\frac{\Delta}{\log \Delta}\right)$ colorable; see [14]. The next theorem follows since $G_{T}(n)$ is $(n-3)$-regular and triangle-free.

Theorem 4.2 For every integer $n \geq 3, \chi\left(G_{T}(n)\right)=O\left(\frac{n}{\log n}\right)$.

We remark that the proof of Theorem 4.1 is constructive, whereas Johansson's proof is probabilistic.

\section{Perfect Matchings of $K_{2 n}$}

Given any graph $G$, let $\mathcal{M}(G)$ be the graph whose vertex set is the set of perfect matchings of $G$, where two vertices of $\mathcal{M}(G)$ are adjacent whenever the symmetric difference of the corresponding perfect matchings is a cycle of length 4 . (See Figure 7 ).

We remark that (contrary to the other graphs we study in this paper) we do not consider geometry in the definition of $\mathcal{M}(G)$. This is because the geometric version of this graph $\mathrm{h}^{(i)}$ is bipartite, as was shown by Hernando et al. [8]. Interestingly, the chromatic number turns out to be quite challenging when geometry is discarded.

We first determine the number of vertices of $\mathcal{M}\left(K_{2 n}\right)$. Let $f(n):=\left|V\left(\mathcal{M}\left(K_{2 n}\right)\right)\right|$ be the number of perfect matchings of $K_{2 n}$. Say $V\left(K_{2 n}\right)=\left\{v_{1}, \ldots, v_{2 n}\right\}$. In every such matching, $v_{1}$ is matched with some vertex in $\left\{v_{2}, \ldots, v_{2 n}\right\}$, and the remaining matching is isomorphic to a perfect matching of $K_{2 n-2}$. Every matching obtained in this way is distinct. Thus

$$
f(n)=(2 n-1) \cdot f(n-1),
$$

(i) In the geometric version of this graph, the vertices are the non-crossing perfect matchings of a set of $2 n$ points in convex position; two of which are adjacent whenever their symmetric difference is a cycle of length four. 


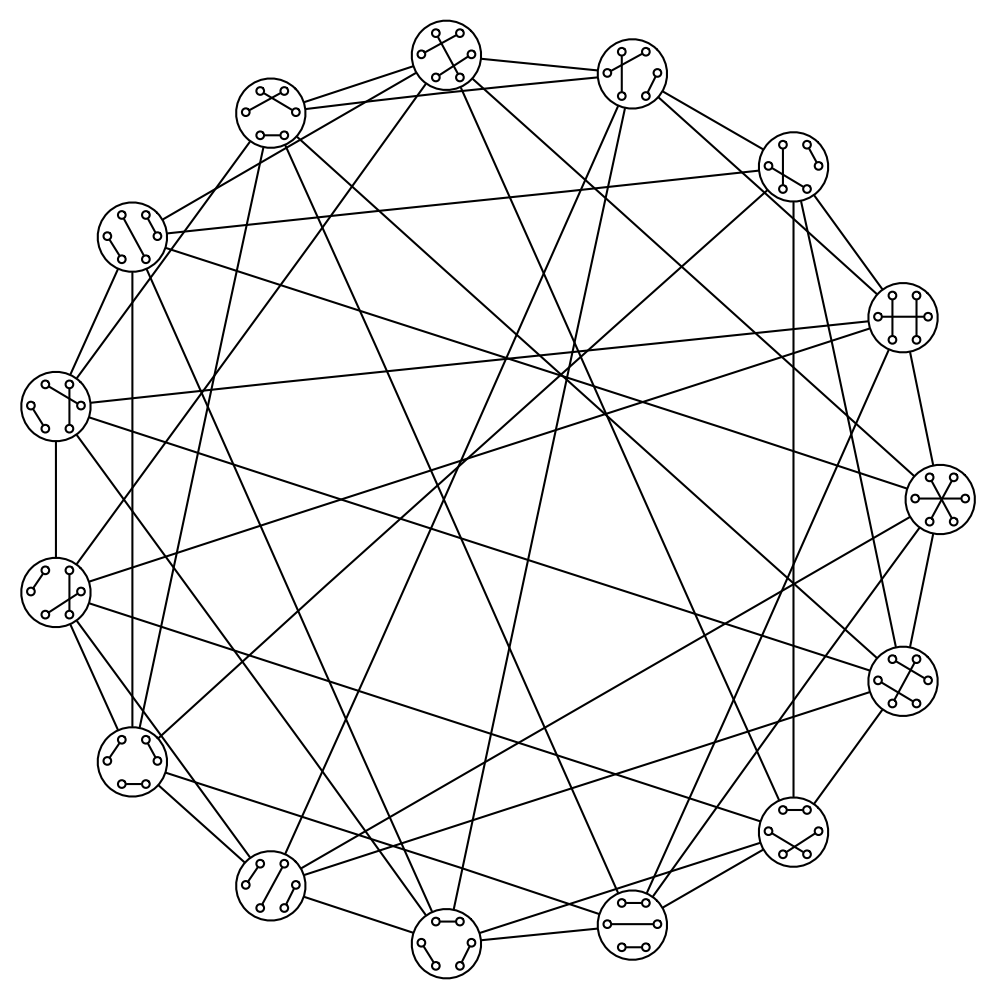

Fig. 7: The graph $\mathcal{M}\left(K_{6}\right)$.

where $f(1)=1$. Hence ${ }^{(\text {ii) }}$

$$
f(n)=(2 n-1) ! !=(2 n-1)(2 n-3)(2 n-5) \ldots 1=\frac{(2 n) !}{2^{n} n !} .
$$

By Stirling's Approximation,

$$
f(n)=\frac{(2 n) !}{2^{n} n !} \approx \sqrt{2}\left(\frac{2 n}{e}\right)^{n} .
$$

Also observe that $\mathcal{M}\left(K_{2 n}\right)$ is $n(n-1)$-regular. Furthermore, the open neighborhood of each vertex of $\mathcal{M}\left(K_{2 n}\right)$ induces a perfect matching; thus $\mathcal{M}\left(K_{2 n}\right)$ has no $K_{4}$ subgraph.

We now give an upper bound on $\chi\left(\mathcal{M}\left(K_{2 n}\right)\right)$. To do so, we use the fact that $\chi\left(\mathcal{M}\left(K_{n, n}\right)\right)=2$. In [8], the flip graph of the non-crossing geometric matchings of a set of $2 n$ points in convex position is shown to be bipartite. This graph is actually a subgraph of $\mathcal{M}\left(K_{n, n}\right)$. Using the same arguments as in [8] we show that $\mathcal{M}\left(K_{n, n}\right)$ is bipartite: Let $\{1, \ldots, n\}$ and $\left\{1^{\prime}, \ldots, n^{\prime}\right\}$ be the color classes of $K_{n, n}$. Then each perfect matching of $\mathcal{M}\left(K_{n, n}\right)$ can be interpreted as a permutation $\sigma$ of $\{1, \ldots, n\}$, where

(ii) Note that the operator !! is called double factorial in the literature. 
$\sigma(i)=j^{\prime}$ if $i$ is matched to $j^{\prime}$. Now, two perfect matchings of $\mathcal{M}\left(K_{n, n}\right)$ are adjacent if and only if their associated permutations differ by a transposition. Thus, these associated permutations have different parity and consequently $\mathcal{M}\left(K_{n, n}\right)$ is bipartite.

Theorem 5.1 For every integer $n \geq 2, \chi\left(\mathcal{M}\left(K_{2 n}\right)\right) \leq 4 n-4$.

Proof: Let $\{1, \ldots, n\}$ and $\left\{1^{\prime}, \ldots, n^{\prime}\right\}$ be the color classes of $K_{n, n}$, and let $\{1, \ldots, 2 n\}$ be the set of vertices of $K_{2 n}$. For every perfect matching $M$ of $K_{2 n}$, let $U(M):=\left\{i \in V\left(K_{2 n}\right) \mid(i, j) \in M\right.$ and $i>$ $j\}$ and $D(M):=\left\{i \in V\left(K_{2 n}\right) \mid(i, j) \in M\right.$ and $\left.i<j\right\}$. Assign to every matching $M$ the number $c(M):=\left(\sum_{i \in D(M)} i\right) \bmod (2 n-2)$. Given two sets $U$ and $D$, let $\mathbb{M}(U, D)$ be the set of matchings $M$ such that $U(M)=U$ and $D(M)=D$. The subgraph $H_{U, D}$ of $\mathcal{M}\left(K_{2 n}\right)$ induced by $\mathbb{M}(U, D)$ is a subgraph of $\mathcal{M}\left(K_{n, n}\right)$, and is thus 2-colorable. Color the vertices of $H_{U, D}$ with colors $c(M)$ and $c^{\prime}(M):=c(M)+2 n-2$.

We show now that if two matchings $M$ and $M^{\prime}$ differ by a flip, then they receive different colors. Two cases arise: Either $U(M)=U\left(M^{\prime}\right)$ and $D(M)=D\left(M^{\prime}\right)$, or for some $i \neq j, U(M)=U\left(M^{\prime}\right)-\{i\}+$ $\{j\}$ and $D(M)=D\left(M^{\prime}\right)-\{j\}+\{i\}$. In the first case, $M$ and $M^{\prime}$ belong to $H_{U(M), D(M)}$ and thus receive different colors. In the second case, $c(M)=c\left(M^{\prime}\right)-j+i$. Since $1 \in D(M) \cap D\left(M^{\prime}\right)$ and $2 n \in U(M) \cap U\left(M^{\prime}\right)$, then $|i-j| \leq 2 n-3$. Therefore $c(M) \neq c\left(M^{\prime}\right)$, and consequently the colors assigned to $M$ and $M^{\prime}$ are different.

Thus we obtain a coloring of $\mathcal{M}\left(K_{2 n}\right)$ using $2(2 n-2)=4 n-4$ colors.

\section{Open Problems}

The most challenging open problems arising from this work are to determine the chromatic numbers of $G_{T}(n)$ and $\mathcal{M}\left(K_{2 n}\right)$. Despite our efforts, we have not been able to obtain non-trivial lower bounds for these graphs. We can formulate the following educated guess on the chromatic number of $\mathcal{M}\left(K_{2 n}\right)$.

Conjecture 6.1 For every integer $n \geq 2, \chi\left(\mathcal{M}\left(K_{2 n}\right)\right)=n+1$.

We have verified this conjecture with the aid of a computer for $n=2,3,4$.

We have studied $\mathcal{M}\left(K_{2 n}\right)$, but it would be very interesting to study $\mathcal{M}(G)$ for other graphs.

There is another flip graph, $\mathcal{M}^{\prime}(G)$, related to $\mathcal{M}(G) . \mathcal{M}^{\prime}(G)$ has as its vertex set the perfect matchings of $G$, where two matchings are now adjacent if and only if their symmetric difference is a cycle of arbitrary length. We have yet to study its chromatic number.

With respect to $\chi\left(G_{T}(n)\right)$, the problem seems far more intriguing since we not only do not have a non-trivial lower bound, but we also believe that our upper bound is far from being tight:

Conjecture 6.2 $\lim _{n \rightarrow \infty} \chi\left(G_{T}(n)\right)=\infty$ and $\chi\left(G_{T}(n)\right)=O(\log (n))$.

Let us mention that we do not have any strong evidence on the conjectured upper bound, and the tight value might be $\Theta(\log \log (n))$ or $\Theta(\operatorname{polylog}(n))$, say, but we have chosen a precise value as more stimulating for research.

It would be interesting to see which techniques will be capable of improving the lower bounds of both of these graphs. It is likely that such techniques will turn out to be useful in determining the chromatic number of other graphs as well. 


\section{References}

[1] P. Bose and F. Hurtado. Flips in planar graphs. Computational Geometry: Theory and Applications 42(1):60-80, 2009.

[2] P. Bose, F. Hurtado, E. Rivera-Campo, and D. R. Wood. Partitions of complete geometric graphs into plane trees. Computational Geometry: Theory and Applications 34(2):116-125, 2006.

[3] R. L. Brooks. On coloring the nodes of a network. Mathematical Proceedings of the Cambridge Philosophical Society 37(2):194-197, 1941.

[4] R. Cummins. Hamilton circuits in tree graphs. IEEE Transactions on Circuit Theory 13:82-90, 1966.

[5] V. Estivill-Castro, M. Noy, and J. Urrutia. On the chromatic number of tree graphs. Discrete Mathematics 223:363-366, 2000.

[6] W. Goddard and H. C. Swart. Distances between graphs under edge operations. Discrete Mathematics 161:121-132, 1996.

[7] P. Hell and J. Nešetřil. Graphs and homomorphisms. Oxford University Press, 2004.

[8] C. Hernando, F. Hurtado, and M. Noy. Graphs of non-crossing perfect matchings. Graphs and Combinatorics 18:517-532, 2002.

[9] M. Houle, F. Hurtado, M. Noy, and E. Rivera-Campo. Graphs of triangulations and perfect matchings. Graphs and Combinatorics 21(3):325-331, 2005.

[10] F. Hurtado and M. Noy. Graph of triangulations of a convex polygon and tree of triangulations. Computational Geometry: Theory and Applications 13(3):179-188, 1999.

[11] C. Lee. The associahedron and the triangulations of the n-gon. European Journal of Combinatorics 10(4):551-560, 1989.

[12] D. E. Lucas. Recreations Mathématiques Volume 2. Gauthiers Villars, Paris 1892.

[13] J. M. Lucas. The rotation graph of binary trees is Hamiltonian. Journal of Algorithms 8(4):503-535, 1987.

[14] M. Molloy and B. Reed. Graph colouring and the probabilistic method. Algorithms and Combinatorics Vol. 23, Springer, 2002.

[15] E. Rivera-Campo and V. Urrutia-Galicia. Hamilton cycles in the path graph of a set of points in convex position. Computational Geometry: Theory and Applications 18(2):65-72, 2001.

[16] D. Sleator, R. Tarjan, and W. Thurston. Rotation distance, triangulations, and hyperbolic geometry. Journal of the American Mathematical Society 1:667-682, 1988.

[17] V. Urrutia-Galicia. Algunas propiedades de gráficas geométricas. Ph.D. thesis, Universidad Autónoma Metroploitana-Iztapalala, México D.F., 2000. 